Provided for non-commercial research and education use. Not for reproduction, distribution or commercial use.

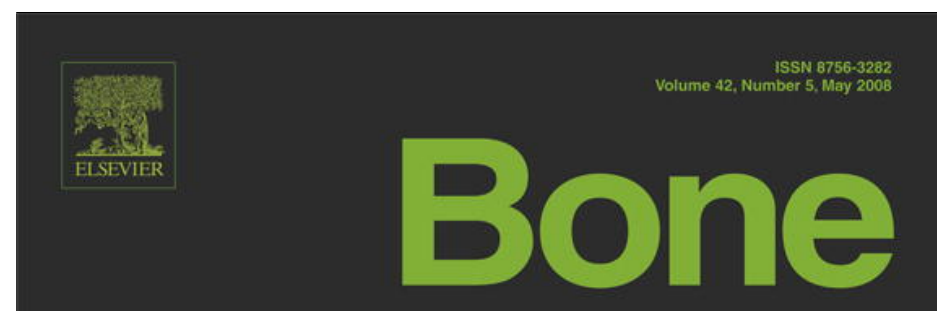

Binding and Uptake of Bisphosphonates

Also in this issue:

- Bisphosphonates and ONJ

- Sympathetic Regulation of Bone Mass - TGF. $\beta 1$ and Osteoporosis

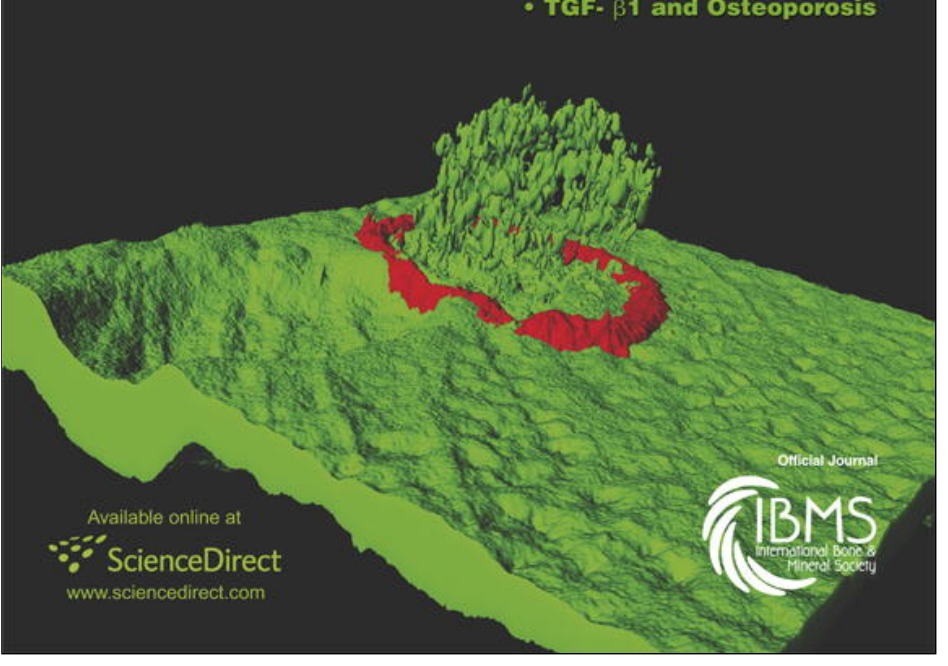

This article appeared in a journal published by Elsevier. The attached copy is furnished to the author for internal non-commercial research and education use, including for instruction at the authors institution and sharing with colleagues.

Other uses, including reproduction and distribution, or selling or licensing copies, or posting to personal, institutional or third party websites are prohibited.

In most cases authors are permitted to post their version of the article (e.g. in Word or Tex form) to their personal website or institutional repository. Authors requiring further information regarding Elsevier's archiving and manuscript policies are encouraged to visit:

http://www.elsevier.com/copyright 
Editorial

\title{
Role of biochemical markers of bone turnover as prognostic indicator of successful osteoporosis therapy
}

\begin{abstract}
Most of the currently available anti-osteoporosis medications promptly and significantly influence the rate of bone turnover. Biochemical markers of bone turnover now provide a high sensitivity to change, allowing the detection of these bone turnover changes within a couple of weeks. Since the anti-fracture efficacy of inhibitors of bone resorption or stimulators of bone formation appears to be largely independent of baseline bone turnover, biochemical markers do not appear to play a significant role in the selection of one particular drug, for an individual patient. However, there are consistent data showing that short-term changes in biochemical markers of bone turnover may be significant predictors of future changes in bone mineral density or fracture reduction, hence suggesting that bone turnover markers play a significant role in the monitoring of anti-osteoporosis therapy.
\end{abstract}

(C) 2008 Elsevier Inc. All rights reserved.

Keywords: Osteoporosis; Monitoring; Treatment; Bone density; Markers of bone turnover

\section{Introduction}

Clinical trials, conducted since the early nineties, have unequivocally shown the ability of several anti-osteoporosis medications to reduce fracture occurrence at various skeletal sites, including but not exhaustively the spine and hip [1]. Whereas the operational definition of osteoporosis is still based on a low bone mineral density (BMD), because low BMD is known to contribute to increased fracture risk, changes in bone mass and density, in response to anti-resorptive therapy, account for only a small portion of the predicted fracture risk reduction [2], a picture which may, however, be significantly changed with the availability of new therapies stimulating bone formation [3] or uncoupling bone formation from bone resorption [4].

During the last decade, biochemical markers of bone turnover (BTM) have been developed, that are more sensitive than conventional ones for detecting abnormalities or changes of bone turnover rate [5]. Increased levels of bone resorption, and short-term changes in BTM, have been shown to predict the risk of fracture, independently of the level of BMD in untreated individuals $[6,7]$.

Dynamic changes in bone turnover, estimated by measurement of bone biochemical markers, such as breakdown products of type I collagen and proteins secreted by osteoblasts and osteoclasts in blood and urine, can also account for a major portion of antifracture efficacy of anti-resorptive or bone-forming agents [8]. Most of the currently marketed anti-osteoporosis medications induce prompt and significant changes in BTM.
Since BTM measurements are non-invasive, fairly inexpensive and can be repeated often, it seems important to provide some guidance about the role BTM can play to identify patients with rapid bone loss, to aid in therapeutic decision-making and to monitor therapeutic efficacy of various treatments.

\section{Baseline BTM as prognosis of future osteoporosis therapy}

One of the major applications of BTM in the field of osteoporosis is to detect high bone turnover in postmenopausal women. This could allow identification of individuals who should be preferentially targeted for prevention or curative therapy, as there is strong evidence that the higher the bone turnover, the greater the future bone loss and/or fracture risk [6].

From a theoretical point of view, it is also conceivable that intervention strategies may differ between patients with various degrees of bone turnover, at the time of diagnosis, i.e. a patient presenting with high rates of bone resorption being optimally treated with anti-resorptive agents, whereas an individual with low remodelling rate expecting greater benefit from a boneforming agent [9].

However, whether the pretreatment (i.e. baseline) BTM concentrations are predictive of a greater response to any of the anti-osteoporotic drugs remains rather controversial.

In a post-hoc analysis of the Fracture Intervention Trial (FIT), the pivotal study based on which alendronate was granted a marketing authorization for the treatment of osteoporosis, alendronate non-spine fracture efficacy was greater among women with high 
pretreatment N-terminal propeptide of type I collagen (P1NP) (Table 1). For osteoporotic women in the lowest tertile of pretreatment P1NP, the alendronate versus placebo relative hazard for non-spine fracture was 0.88 (95\% CI 0.65-1.21) compared to a relative hazard of 0.54 (95\% CI $0.39-0.74)$ among those in the highest tertile of P1NP. Similar results were observed among women without osteoporosis at baseline.

However, the relationship between tertile of bone-specific alkaline phosphatase (BALP) or C-terminal cross-linked telopeptide of type I collagen (sCTX) and the risk of non-spine fracture did not reach statistical significance. There was no relationship between pretreatment P1NP, BALP or sCTX and alendronate efficacy or incident spine fracture among osteoporotic women [10]. Based on the assumption that alendronate may reduce incident non-vertebral fracture to a greater degree in non-osteoporotic women, with high compared to low bone turnover, a recent pharmacoeconomic study (Markov model) concluded that measurement of BTM has the potential to identify a subset of postmenopausal women (top BTM quartile), without osteoporosis by BMD criteria, for whom alendronate therapy to prevent fracture is cost-effective (costs per QALY gained at 34,000 USD and 50,000 USD for women age 70 with high bone turnover and femoral neck BMD T-score of -2.0 and -1.5 respectively) [11].

A similar analysis of the risedronate phase III clinical programmes used the excretion of urinary deoxypyridinoline (DPD) as an index of pretreatment bone resorption rates. During the first year of treatment, women with high DPD (above premenopausal normative median) gained lumbar spine BMD at a faster rate than patients with low DPD. The risedronate-mediated reduction in the incidence of vertebral fractures was independent of baseline DPD. However, the number needed-to-treat (NNT) to avoid one vertebral fracture at 12 months was 15 in the group of patients with high DPD and 25 in patients with low DPD, an observation which is not unexpected, based on the influence of the prevalent absolute risk on NNT calculation [12]. The authors concluded that although the reduction in overall fracture risk seems to occur independent of baseline bone turnover, patient stratification by pretreatment bone resorption rate seems to make some sense from a pharmacoeconomic point of view $[9,12]$.

Little is known about how the baseline levels of BTM relate to the densitometric response or the anti-fracture efficacy of anabolic therapy in postmenopausal women. A post-hoc analysis of the Fracture Prevention Trial (FPT), the study which demonstrated vertebral and non-vertebral fracture reduction with the recombinant human parathyroid hormone rhPTH (1-34) (teriparatide), assessed the correlation between BTM and BMD responses. Bone turnover status at baseline correlated significantly with subsequent BMD responses. The highest correlation was found with the changes in lumbar BMD observed after 18 months for teriparatide treatment. All correlations with BTM were significant and positive and from largest to smallest, with P1NP, urinary N-terminal telopeptide of type I collagen (uNTX), serum carboxy-terminal extension peptide of procollagen type I (P1CP), BALP and DPD. For femoral neck BMD changes, the coefficients of correlation were of a much lower magnitude and remained significant only for UNTX and DPD [13]. In another study assessing the relationship between levels of baseline turnover before PTH $(1-84)$ and the subsequent changes in area and volumetric BMD, higher baseline P1NP levels were associated with greater increase in areal BMD. Each standard deviation (SD) increase in baseline P1NP was associated with an additional $1.7 \%$ increase $(95 \%$ CI $0.5-3.0)$ in spine BMD (assessed by dual-energy X-ray absorpotiometry [DXA]) and a $1.2 \%$ increase $(95 \%$ CI $0.4-2.0)$ in hip DXA BMD, after 12 months of PTH. Higher baseline BALP or sCTX levels were also associated with greater 1-year increases in hip and spine DXA BMD respectively. Neither baseline P1NP nor BALP were significantly associated with 1-year changes in volumetric BMD (by quantitative computerised tomography [QCT]). Higher baseline sCTX levels were associated with greater 1-year increases in trabecular spine and cortical hip BMD QCT [14]. The association between pretreatment BTM concentration and the absolute and relative risks of vertebral and non-vertebral fractures (FPT of teriparatide) was evaluated after adjusting for baseline femoral neck BMD, number of prevalent fractures and age. Higher pretreatment concentrations of BALP, P1NP, NTX and DPD were associated with a greater risk of fracture. Teriparatide significantly reduced the risk, regardless of pretreatment bone turnover. For each BTM, the absolute risk of an osteoporotic fracture was greatest in the highest tertile, while the relative risk was not associated with baseline bone turnover. Hence, the NNT to prevent a new osteoporotic fracture decreased as the BTM concentration increased (from 10 to 7 for low to high P1NP tertile) [15]. The authors concluded that teriparatide-mediated relative fracture risk reduction was independent of pretreatment bone turnover, which is supported by the absence of correlation between baseline P1CP, DPD or UNTX and changes in structural and dynamic bone parameters analysed from transiliac biopsy specimens obtained during the same FPT [16].

Table 1

Summary of biochemical markers used in studies having assessed their role as predictor of response to anti-osteoporotic drug

\begin{tabular}{ll}
\hline Treatment & Biochemical marker studied \\
\hline Alendronate $[10,17,18,19,20,21]$ & Formation: BALP, PINP, PICP, OC \\
Risedronate $[12,22]$ & Resorption: sCTX, uNTX, DPD \\
Teriparatide $[13,14,15,33]$ & Resorption: uDPD, uCTX, uNTX, \\
Raloxifene $[30,31,32]$ & Formation: BALP, PICP, PINP Fracture, BMD \\
& Resorption: uDPD, uNTX, sCTX \\
& Formation: BALP, PINP, OC Fracture, BMD \\
\hline
\end{tabular}




\section{Early changes in BTM to monitor anti-osteoporosis therapy}

Similar to most chronic diseases, monitoring the efficacy of treatment of osteoporosis is a challenge. In contrast to BMD, which typically changes in response to therapy less than $2-5 \%$ per year, or a maximum of 3\% in 3-6 months, most of osteoporosis therapies act by reducing or increasing individual BTM levels or their ratios by $30-200 \%$ within 3-6 months. Even though BMD measurement is much more precise than BTM, it is still easier to detect a $50 \%$ change with a BTM than a $3 \%$ change in BMD with a DXA, after 3-6 months of therapy [8]. Generally, the ratio of signal to noise during the first year of treatment is better for BTM than it is for BMD.

In early postmenopausal women receiving alendronate in the prevention of osteoporosis (Early Postmenopausal Intervention Cohort - EPIC study), change from baseline at month 6 in uNTX or osteocalcin (OC) correlated with change from baseline at month 24 in spine, hip and total body BMD. This corresponded to a 4 - to 5-fold greater increase at month 24 in BMD in the tertile with the greatest decrease at month 6 in NTX or OC compared to the tertile with the least decrease in the BTM. In this study, a patient with a decrease of $40 \%$ for NTX or $20 \%$ for OC at month 6 had a $92 \%$ probability of a 2 -year positive response in spine $\mathrm{BMD}$. In contrast, the poor specificity and negative predictive value of these percentual cut-offs of BTM changes implied that a change in NTX or OC above the cut-point was a poor predictor of bone loss during alendronate treatment [17]. In a smaller cohort of French osteoporotic women, the authors claimed, from observation of changes in BALP, OC, P1CP, P1NP, sCTX, uNTX, total and free DPD after 4 months of alendronate, that SCTX, and to a lesser extend, uNTX, were the best predictors of a significant gain in spine BMD after 1 year of therapy [18]. Early changes of uNTX (3 months) were also shown to be good predictors of 12-month lumbar spine BMD response to alendronate in Korean postmenopausal women with osteoporosis [19]. Similar features were reported for 6-month decreases in UNTX and DPD, as surrogate for 2.5-year increases in hip, trochanter, spine and total BMD in elderly US women [20]. The data from the FIT were used to assess the relationship between changes in bone turnover after 1 year of alendronate and subsequent hip, non-spine and spine fracture risk. Each 1SD reduction in 1-year change in BALP was associated with fewer spine (relative hazard [RH] 0.74, 95\% CI 0.63-0.87), non-spine (RH $0.89,95 \%$ CI $0.78-1.00$ ) and hip fracture (RH $0.61,95 \%$ CI $0.46-0.78)$. Alendronate-treated women with at least a 30\% reduction in BALP had a lower risk of non-spine $(\mathrm{RH}$ $0.72,95 \%$ CI $0.55-0.92$ ) and hip fracture (RH $0.26,95 \% \mathrm{CI}$ $0.08-0.83$ ) relative to those with reduction $<30 \%$. The authors concluded that the association between early reduction in bone turnover and long-term fracture reduction during alendronate treatment was at least as strong as that observed with 1-year changes in BMD [21].

Changes in the level of biochemical markers of bone resorption with risedronate treatment for osteoporosis were also examined as a surrogate for the decrease in fracture risk, in osteoporotic patients from the risedronate vertebral fracture trial. The reduction in urinary $\mathrm{sCTX}$ (median, 60\%) and uNTX $(51 \%)$ at 3-6 months with risedronate therapy were significantly associated $(p<0.05)$ with the reduction in vertebral fracture risk ( $75 \%$ over 1 year and $50 \%$ over 3 years). The changes in both sCTX and uNTX accounted for approximately one-half (sCTX, $55 \%$; uNTX, 49\%) of the first year and approximately two-thirds (sCTX, 67\%; uNTX, 66\%) over 3 years compared with placebo. The changes in SCTX and uNTX accounted for $77 \%$ and $54 \%$, respectively, of risedronate effect in reducing the risk of nonvertebral fractures over 3 years compared with placebo. The relationships between vertebral fracture risk and changes from baseline in sCTX and uNTX were not linear $(p<0.05)$. In the original publication [22] below a decrease of $55-60 \%$ for sCTX and $35-40 \%$ for uNTX, the authors concluded that the decrease in bone resorption in patients taking risedronate accounts for a large proportion of the reduction in fracture risk but that there was little further improvement in anti-fracture efficacy below a decrease of $55-60 \%$ for SCTX and $35-40 \%$ for uNTX. [22]. The data from this study were recently reanalysed by another team of statisticians. The findings of the reanalysis still support the original conclusion that there is a level of bone resorption below which there is no fracture benefit, but this was only significant for sCTX at a level of 51\% decrease [23].

Whereas the relationship between BTM and BMD or fracture reduction, in individual patients, has not yet been published, for oral or intravenous ibandronate, the course of the development of this bisphosphonate strongly support the value of BTM as a surrogate for BMD or fracture assessment in osteoporosis. The earliest studies conducted with ibandronate, using suboptimal doses ( 1 and $0.5 \mathrm{mg}$ IV given once every 3 months) resulted in marginal decreases in biochemical markers of bone resorption and formation, which eventually translated into small increases in spine BMD and insufficient magnitude of fracture reduction to achieve statistical significance [24]. By contrast, with continuous (2.5 mg daily) or intermittent $(20 \mathrm{mg}$ every other days for 12 doses every 3 months) oral doses which were linked to a significant reduction in spinal fractures, similar to that seen with alendronate or risedronate, the rate of bone turnover was reduced by $50-60 \%$, a magnitude also within the range observed with the oral efficacious oral bisphosphonates [25]. For further clinical development of ibandronate, the role of BTM, as predictor of efficacy was even emphasised. Actually, a pharmacokinetic-pharmacodynamic model, accurately describing the dose-dependent predicted urinary excretion of CTX, has surrogate of clinical efficacy, was used to select the appropriate once-monthly dose of ibandronate [26]. Clinical studies, showing the non-inferiority and/or superiority of the $150 \mathrm{mg}$ monthly oral regimen, on $\mathrm{BMD}$, as compared to the daily 2.5 dose, the dose which previously demonstrated anti-fracture efficacy [27] confirmed, a posteriori, the interest of a pharmacostatistical model based on BTM changes to predict the effect of a particular dosage of ibandronate on hard end-points [28].

Data from randomized clinical studies of ibandronate, given orally or intravenously, at different doses and for variable time intervals to women with osteoporosis were examined to explore the relationship between intermittent bisphosphonate therapy, changes in bone resorption and fracture risk. The magnitude of the reduction of the rate of bone resorption at the end of the 
drug-free interval rather than its fluctuation pattern after bisphosphonate administration determines anti-fracture efficacy, provided that these fluctuations occur within the premenopausal range [29]. From these results, the authors concluded that prolongation of the drug-free interval should be compensated by a dose higher than the cumulative daily dose [29].

In the Multiple Outcomes of Raloxifene Evaluation (MORE) trial, which demonstrated vertebral fracture reduction with raloxifene, changes in OC and BALP, after 6 and 12 months, were significantly related to future risk of vertebral fracture, also after adjusting for baseline vertebral status and BMD. For a decrease of $9.3 \mathrm{pg} / \mathrm{l}$ in OC after 1 year raloxifene therapy, the odds ratio (OR) for a new vertebral fracture during 3 years was 0.69 (95\% CI, 0.54-0.88). Similarly, for a decrease of $5.91 \mu \mathrm{g} / \mathrm{l}$ in BALP, OR was $0.75(95 \% \mathrm{CI}, 0.62-0.92)$ [30]. After adjustment of each significant baseline variable, in logistic regression models using 1-year percent changes in BMD and bone turnover and relevant baseline demographics to predict the risk of vertebral fracture with raloxifene therapy at 3 years, the percent changes in $\mathrm{OC}$ was better able to predict the reduction in fracture risk than the percent changes in femoral neck BMD [31]. When logistic regression analysis models were used to evaluate the relationship between the changes at 1 year in P1NP, OC, BALP, and urinary CTX and the risk of new vertebral fractures at 3 years, in the same study, a 1-year decrease in P1NP, BALP or OC but not urinary CTX was again predictive of the 3-year vertebral fracture risk reduction with raloxifene. The decrease in P1NP at 1 year accounted for $28 \%$ of the total reduction in vertebral fracture risk [32].

Among all studied BTM in the teriparatide FPT, increases in P1CP at 1 month and P1NP at 3 months correlated best with increases in lumbar spine BMD at 18 months. The relationship between these two BTM and the lumbar spine BMD response, was stronger than the corresponding relationship for the femoral neck BMD response [13]. Greater short-term changes in P1NP during PTH (1-84) treatment were associated with greater 1-year increase in spine and hip BMD among postmenopausal osteoporotic women. Each SD increase in the 3-month change of P1NP was associated with a $21 \%$ greater increase in QCT spine trabecular BMD [14]. When evaluating associations between early changes in biochemical markers and structural and dynamic bone parameters during teriparatide treatment, changes in BALP at 1 month correlated with changes at 22 months in two-dimensional wall thickness, trabecular bone volume, marrow star volume and trabecular thickness while changes in P1CP at 1 month correlated with change in wall thickness. Whereas no data are currently available to claim that short-term changes in BTM during teriparatide are predictive of future fracture reduction, the correlation of biochemical markers of bone formation (but not resorption) with improvements of bone structure indirectly support this hypothesis [16]. Based on these results, an algorithm for using P1NP to monitor treatment of patients with teriparatide was further developed [33]. Patients with P1NP increases $>10 \mu \mathrm{g} / 1$ after $1-3$ months, were considered as responders while it was recommended that patients with P1NP increases $<10 \mu \mathrm{g} / 1$ should be assessed for adherence, teriparatide administration and storage technique and for the presence of medical conditions that might limit their therapeutic response to teriparatide [33].

\section{Conclusion}

In conclusion, most of the currently available pharmacological treatments for osteoporosis promptly and significantly impact on the rate of bone turnover. BTM do not appear to be a significant determinant in the selection of a particular antiosteoporosis drug. With the exception of a single and equivocal study with alendronate, the anti-fracture efficacy of anti-osteoporosis agents appears to be largely independent of baseline bone turnover. However, short-term changes in BTM reflecting bone resorption (raloxifene, bisphosphonates) or bone formation (raloxifene, teriparatide, (1-84) parathyroid hormone) have been consistently shown to predict future BMD changes (teriparatide, parathyroid hormone) or long-term fracture reduction (alendronate, risedronate, raloxifene), strongly suggesting that BTM could play a significant role in the monitoring of antiosteoporosis therapy. Further research should be stimulated to assess the relevance of these findings to the care of individual patients, i.e. how to define a response to a specific treatment based on the short-term changes observed in BTM.

\section{References}

[1] Boonen S, Body JJ, Boutsen Y, Devogelaer JP, Goemaere S, Kaufman JM, et al. Evidence-based guidelines for the treatment of postmenopausal osteoporosis: a consensus document of the Belgian Bone Club. Osteoporos Int 2005;16:239-54.

[2] Delmas PD, Seeman E. Changes in bone mineral density explain little of the reduction in vertebral or nonvertebral fracture risk with anti-resorptive therapy. Bone 2004;34:599-604.

[3] Chen P, Miller PD, Delmas PD, Misurski DA, Krege JH. Change in lumbar spine BMD and vertebral fracture risk reduction in teriparatide-treated postmenopausal women with osteoporosis. J Bone Miner Res 2006;21: 1785-90.

[4] Bruyère O, Roux C, Detilleux J, Slosman DO, Spector TD, Fardellone P, et al. Relation between bone mineral density changes and fracture risk reduction in patients treated with strontium ranelate. J Clin Endocrinol Metab 2007;92:3076-81.

[5] Delmas PD, Eastell R, Garnero P, Seibel MJ, Stepan J, Committee of Scientific Advisors of the International Osteoporosis Foundation. The use of biochemical markers of bone turnover in osteoporosis. Committee of Scientific Advisors of the International Osteoporosis Foundation. Osteoporos Int 2000;11:S2-S17.

[6] Garnero P, Hausherr E, Chapuy MC, Marcelli C, Grandjean H, Muller C, et al. Markers of one resorption predict hip fracture in elderly women: the EPIDOS Prospective Study. J Bone Miner Res 1996;11:1531-8.

[7] Bruyère O, Collette J, Delmas P, Rouillon A, Roux C, Seidel L, et al. Interest of biochemical markers of bone turnover for long-term prediction of new vertebral fracture in postmenopausal osteoporotic women. Maturitas 2003;44:259-65

[8] Srivastava AK, Vliet EL, Lewiecki EM, Maricic M, Abdelmalek A, Gluck $\mathrm{O}$, et al. Clinical use of serum and urine bone markers in the management of osteoporosis. Curr Med Res Opin 2005;21:1015-26.

[9] Seibel MJ. Biochemical markers of bone turnover part II: clinical applications in the management of osteoporosis. Clin Biochem Rev 2006;27:123-38.

[10] Bauer DC, Gamero P, Hochberg MC, Santora A, Delmas P, Ewing SK, et al, for the Fracture Intervention Research Group. Pretreatment levels of bone turnover and antifracture efficacy of alendronate: the fracture intervention trial. J Bone Miner Res 2006;21:292-9. 
[11] Schousboe JT, Bauer DC, Nyman JA, Kane RL, Melton LJ, Ensrud KE. Potential for bone turnover markers to costs-effectively identify and select post-menopausal osteopenic women at high risk of facture for bisphosphonate therapy. Osteoporos Int 2007;18:201-10.

[12] Seibel MJ, Naganathan V, Barton I, Grauer A. Relationship between pretreatment bone resorption and vertebral fracture incidence in postmenopausal osteoporotic women treated with risedronate. J Bone Miner Res 2004;19:323-9

[13] Chen P, Satterwhite JH, Licata AA, Lewiecki EM, Sipos AA, Misursk $\mathrm{DM}$, et al. Early changes in biochemical markers of bone formation predict BMD response to teriparatide in postmenopausal women with osteoporosis. J Bone Miner Res 2005;20:962-70.

[14] Bauer DC, Garnero P, Bilezikjan JP, Greenspan SL, Ensrud KE, Rosen CJ, et al. Short-term changes in bone turnover markers and bone mineral density response to parathyroid hormone in postmenopausal women with osteoporosis. J Clin Endocrinol Metab 2006;91:1370-5.

[15] Delmas PD, Licata AA, Reginster JY, Crans GG, Chen P, Misurski DA, et al. Fracture risk reduction during treatment with teriparatide is dependent of pretreatment bone turnover. Bone 2006;39:237-43.

[16] Dobing H, Sipos A, Jiang Y, Fahrleitner-Pammer A, Ste-Marie LG, Gallagher JC, et al. Early changes in biochemical markers of bone formation correlate with improvements in bone structure during teriparatide therapy. J Clin Endocrinol Metab 2005;90:3970-7.

[17] Rayn P, Hosking D, Thompson D, Cizza G, Wasnich RD, McClung M, et al Monitoring of alendronate treatment and prediction of effect on bone mass by biochemical markers in the early postmenopausal intervention cohort study. J Clin Endocrinol Metab 1999;84:2363-8.

[18] Fink E, Cormier C, Steinmetz P, kindermans C, Le Bouc Y, Souberbielle JC. Differences in the capacity of several biochemical bone markers to assess high bone turnover in early menopause and response to alendronate therapy. Osteoporos Int 2000;11:295-303.

[19] Kim SW, Park DJ, Park KS, Kim SY, Cho BY, Lee HK, et al. Early changes in biochemical markers of bone turnover predict bone mineral density response to antiresorptive therapy in Korean postmenopausal women with osteoporosis. Endocr J 2005;52:667-74.

[20] Greenspan SL, parker RA, Ferguson L, Rosen HN, Maitland-Ramsey L, Karpf DB. Early changes in biochemical markers of bone turnover predict the long-term response to alendronate therapy in representative elderly women: a randomized clinical trial. J Bone Miner Res 1998;13: 1431-8.

[21] Bauer DC, Black DM, Garnero P, Hochberg M, Ott S, Orloff J, et al. Change in bone turnover and hip, non-spine, and vertebral fracture in alendronate-treated women: the fracture intervention trial. J Bone Miner Res 2004;19:1250-8.

[22] Eastell R, Barton I, Hannon RA, Chines A, Garnero P, Delmas PD. Relationship of early changes in bone resorption to the reduction in fracture risk with risedronate. J Bone Miner Res 2003;18:1051-6.

[23] Eastell R, Hannon RA, Garnero P, Campbell MJ, Delmas PD. Relationship of early changes in bone resorption to the reduction in fracture risk with risedronate: review of statistical analysis. J Bone Miner Res 2007;22: 1656-60.

[24] Recker R, Stakkestad JA, Chesnut CH, Christiansen C, Skag A, Hoiseth A et al. Insufficiently dosed intravenous ibandronate injections are associated with suboptimal antifracture efficacy in postmenopausal osteoporosis. Bone 2004;34:890-9
[25] Delmas PD, Recker RR, Chesnut CH, Skag A, Stakkestad JA, Emkey R, et al. Daily and intermittent oral ibandronate normalize bone turnover and provide significant reduction in vertebral fracture risk: results from the BONE study. Osteoporos Int 2004;15:792-8.

[26] Reginster JY, Gieschke R. Clinical utility of a pharmacostatistical model for ibandronate in postmenopausal osteoporosis. Curr Drug Metab 2006;7: 827-36.

[27] Reginster JY, Adami S, Lakatos P, Greenwald M, Stepan JJ, Silverman SL et al. Efficacy and tolerability of once-monthly oral ibandronate in postmenopausal osteoporosis: 2 year results from the MOBILE study. Ann Rheum Dis 2006;65:654-61.

[28] Reginster JY, Felsenberg D, Cooper C, Stakkestad JA, Miller PD, Kendler DL, et al. A new concept for bisphosphonate therapy: a rationale for the development of monthly oral dosing of ibandronate. Osteoporos Int 2006;17:159-66.

[29] Papapoulos SE, Schimmer RC. Changes in bone remodelling and antifracture efficacy of intermittent bisphosphonate therapy:implications from clinical studies with ibandronate. Ann Rheum Dis 2007;66:853-8.

[30] Bjarnason NH, Sarkar S, Duong T, Mitlak B, Delmas PD, Christiansen C. Six and twelve month changes in bone turnover are related to reduction in vertebral fracture risk during 3 years of raloxifene treatment in postmenopausal osteoporosis. Osteoporos Int 2001;12:922-30.

[31] Sarkar S, Reginster JY, Crans GG, Diez-Perez A, Pinette KV, Delmas PD Relationship between changes in biochemical markers of bone turnover and BMD to predict vertebral fracture risk. J Bone Miner Res 2004;19: 394-401.

[32] Reginster JY, Sarkar S, Zegels B, Henrotin Y, Bryere O, Agnusdei D, et al. Reduction in P1NP, a marker of bone metabolism, with raloxifene treatment and its relationship with vertebral fracture risk. Bone 2004;34: 344-51.

[33] Eastell R, Krege JH, Chen P, Glass EV, Reginster JY. Development of an algorithm for using P1NP to monitor treatment of patients with teriparatide. Curr Med Res Opin 2006;22:61-6.

Jean-Yves Reginster* Julien Collette

Audrey Neuprez

Brigitte Zegels

Rita Deroisy

Olivier Bruyere

Department of Public Health, Epidemiology and Health Economics, CHU Sart Tilman, University of Liège,

Liège, Belgium

*Corresponding author. Bone and Cartilage Metabolism Research Unit, CHU Centre-Ville, Policliniques L. BRULL, Quai Godefroid Kurth 45 (9ème étage), 4020 Liège, Belgium.

Fax: +32 4270.32 .53

E-mail address: jyreginster@ulg.ac.be (J.-Y. Reginster).

19 November 2007 